\title{
Vascularization for cooling and reduced thermal stresses
}

\author{
E. Cetkin ${ }^{\mathrm{a}}$, S. Lorente ${ }^{\mathrm{b}}$, A. Bejan ${ }^{\mathrm{c}, *}$ \\ a Izmir Institute of Technology, Department of Mechanical Engineering, Urla, Izmir 35430, Turkey \\ ${ }^{\mathrm{b}}$ Université de Toulouse, UPS, INSA, 135, avenue de Rangueil, F-31 077 Toulouse Cedex 04, France \\ ${ }^{\mathrm{c}}$ Duke University, Department of Mechanical Engineering and Materials Science, Durham, NC 27708-0300, USA
}

\section{A R T I C L E I N F O}

\section{Article history:}

Received 6 May 2014

Received in revised form 12 September

2014

Accepted 12 September 2014

Available online 19 October 2014

\section{Keywords:}

Constructal

Vascular

Cooling

Strength

Thermal stresses

Thermal expansion

\begin{abstract}
A B S T R A C T
This paper documents the effect of thermal expansion on a vascularized plate that is heated and loaded mechanically. Vascular cooling channels embedded in a circular plate provide cooling and mechanical strength. The coolant enters the plate from the center and leaves after it cools the plate to an allowable temperature limit. The mechanical strength of the plate decreases because of the embedded cooling channels. However, cooling the plate under an allowable temperature level decreases the thermal stresses. The mechanical strength of the plate which is heated and loaded mechanically at the same time can be increased by inserting cooling channels in it. The mechanical and thermofluid behavior of a vascularized plate was simulated numerically. The cooling channel configurations that provide the smallest peak temperature and von Mises stress are documented. There is one cooling channel configuration that is the best for the given set of boundary conditions and constraints; however, there is no single configuration that is best for all conditions.
\end{abstract}

(c) 2014 Elsevier Ltd. All rights reserved.

\section{Introduction}

Advanced technologies require smart capabilities with compactness and light weight for structures that work under significant heat fluxes and mechanical loads. The mechanical strength can be increased by adding more material, however this is not desirable because of the weight increase. In addition, the structure should be able to resist intense heat fluxes. The design challenge is to increase simultaneously the mechanical strength and the cooling performance while decreasing the weight.

This design challenge makes vascularized structure an attractive design for advanced technologies. Smart capabilities can be built into a material by embedding vascular channels in it [1-8]. The vascular channels increase the mechanical strength if the material removed to make room for the channels is placed around the channels, i.e. if the amount of solid material is fixed [8]. In addition, the peak temperature can be kept under an allowable limit by circulating coolant through the vascular channels [7-10]. In summary, the material should be placed where it is needed the most, i.e. where it decreases the resistances to the flow of heat, fluid and stress. The design should be free to vary, in accord with the Constructal design method [1]. The current literature details the application of this method to smart structures [1-8,11-13] and thermofluid design [14-28]. In a few instances, mechanical

\footnotetext{
* Corresponding author.
}

strength was used as the design objective [29-31]. However, the effect of thermal expansion on stresses has been overlooked in the literature.

If the heating effect is small enough, and the mechanical strength of a material is governed by the mechanical loading then the effect of thermal expansion can be neglected. However, the effect of thermal expansion is an essential design parameter for the vehicles and parts that work under great heat fluxes and mechanical loads such as turbine blades, engine parts, electronics and aerospace applications. This paper documents the effect of thermal expansion on the strength and thermal performance of a vascularized plate. The search is for architectures perform the best by facilitating strength and cooling at the same time. The emerging structures consist of embedded radial and tree-shaped cooling channels.

\section{Model}

Consider a circular plate with embedded cooling channels (Fig. 1). The diameter of the circular domain is $D$, and the plate thickness is $H=0.1 D$. The plate is heated and loaded uniformly on its bottom surface. The total volume of the structure and the flow volume are fixed. The flow volume is $3 \%$ of the total volume. The coolant enters from the center of the plate and leaves from the rim. The flow is driven by the pressure difference maintained between the inlet and outlet ports. 


\begin{tabular}{|c|c|c|c|}
\hline \multicolumn{4}{|c|}{ Nomenclature } \\
\hline$c_{P}$ & specific heat at constant pressure, $\mathrm{J} \mathrm{kg}^{-1} \mathrm{~K}^{-1}$ & $\lambda$ & fluid thermal diffusivity, $\mathrm{m}^{2} \mathrm{~s}^{-1}$ \\
\hline$D$ & plate diameter, $\mathrm{m}$ & $\eta$ & number of the cooling channels connected to the \\
\hline$d$ & diameter of cooling channels, $\mathrm{m}$ & & distributing channel \\
\hline E & elasticity modulus, $\mathrm{N} \mathrm{m}^{-2}$ & $\sigma$ & normal stress, $\mathrm{N} \mathrm{m}^{-2}$ \\
\hline$H$ & plate thickness, m & $\tau$ & shear stress, $\mathrm{N} \mathrm{m}^{-2}$ \\
\hline$k$ & thermal conductivity, $\mathrm{W} \mathrm{m}^{-1} \mathrm{~K}^{-1}$ & $\varphi$ & shear strain \\
\hline$n$ & normal direction & & \\
\hline$P$ & pressure, $\mathrm{N} \mathrm{m}^{-2}$ & \multicolumn{2}{|c|}{ Subscripts } \\
\hline$P_{l}$ & mechanical load, $\mathrm{N} \mathrm{m}^{-2}$ & 0 & main distributing channel \\
\hline$P_{\text {in }}$ & inlet pressure, $\mathrm{N} \mathrm{m}^{-2}$ & 1 & first level of cooling channel \\
\hline $\mathrm{Pr}$ & Prandtl number & 2 & second level of cooling channel \\
\hline$q^{\prime \prime}$ & imposed heat flux, $\mathrm{W} \mathrm{m}^{-2}$ & $f$ & fluid \\
\hline$r_{x, y, z}$ & displacements, m & in & inlet \\
\hline$T$ & temperature, $\mathrm{K}$ & $m$ & mechanical \\
\hline$u, v, w$ & velocity components, $\mathrm{m} \mathrm{s}^{-1}$ & $\max$ & maximum \\
\hline$x, y, z$ & coordinates, m & ref & reference \\
\hline & & $S$ & solid \\
\hline Greek & nbols & th & thermal \\
\hline$\alpha$ & thermal expansion coefficient, $\mathrm{K}^{-1}$ & out & outlet \\
\hline$\gamma$ & Poisson ratio & $x, y, z$ & coordinates \\
\hline$\varepsilon$ & strain & & \\
\hline$\mu$ & dynamic viscosity, $\mathrm{kg} \mathrm{m}^{-1} \mathrm{~s}^{-1}$ & \multicolumn{2}{|c|}{ Superscript } \\
\hline$v$ & kinematic viscosity, $\mathrm{m}^{2} \mathrm{~s}^{-1}$ & $\sim$ & dimensionless \\
\hline$\rho$ & density, $\mathrm{kg} \mathrm{m}^{-3}$ & & \\
\hline
\end{tabular}

The fluid flow is governed by the mass conservation and momentum equations for incompressible steady flow

$\frac{\partial u}{\partial x}+\frac{\partial v}{\partial y}+\frac{\partial w}{\partial z}=0$

$u \frac{\partial u}{\partial x}+v \frac{\partial u}{\partial y}+w \frac{\partial u}{\partial z}=-\frac{1}{\rho} \frac{\partial P}{\partial x}+v \nabla^{2} u$

$u \frac{\partial v}{\partial x}+v \frac{\partial v}{\partial y}+w \frac{\partial v}{\partial z}=-\frac{1}{\rho} \frac{\partial P}{\partial y}+v \nabla^{2} v$

$u \frac{\partial w}{\partial x}+v \frac{\partial w}{\partial y}+w \frac{\partial w}{\partial z}=-\frac{1}{\rho} \frac{\partial P}{\partial z}+v \nabla^{2} w$

Here $\nabla^{2}=\partial^{2} / \partial x^{2}+\partial^{2} / \partial y^{2}+\partial^{2} / \partial z^{2}$; furthermore $x, y$ and $z$ are the spatial coordinates, $u, v$ and $w$ are the velocity components corresponding to these coordinates, and $P, v$ and $\rho$ are the pressure, kinematic viscosity and fluid density. The fluid is single phase with constant properties.

The temperature distribution inside the flow channels is found by solving the energy equation

$\rho c_{P}\left(u \frac{\partial T}{\partial x}+v \frac{\partial T}{\partial y}+w \frac{\partial T}{\partial z}\right)=k_{f} \nabla^{2} T$

where $c_{P}$ is the specific heat at constant pressure of the fluid, $T$ is the temperature, and $k_{f}$ is the fluid thermal conductivity. The continuity of heat flux between the solid and fluid interfaces requires

$k_{s} \frac{\partial T}{\partial n}=k_{f} \frac{\partial T}{\partial n}$

where $n$ is the vector normal to the fluid-solid interface. The temperature distribution inside the solid is obtained by solving $\nabla^{2} T=0$.

The solid plate is isotropic with elastic deformations that are small when compared with the dimensions $D$ and $H$. There are no volumetric forces such as weight or acceleration. With these simplifications in mind, the momentum equations and generalized Hooke's law become $\frac{\partial \sigma_{x x}}{\partial x}+\frac{\partial \tau_{y x}}{\partial y}+\frac{\partial \tau_{z x}}{\partial z}=0$

$\frac{\partial \tau_{x y}}{\partial x}+\frac{\partial \sigma_{y y}}{\partial y}+\frac{\partial \tau_{z y}}{\partial z}=0$

$\frac{\partial \tau_{x z}}{\partial x}+\frac{\partial \tau_{y z}}{\partial y}+\frac{\partial \sigma_{z z}}{\partial z}=0$

$$
\left[\begin{array}{c}
\sigma_{x x} \\
\sigma_{y y} \\
\sigma_{z z} \\
\tau_{y z} \\
\tau_{z x} \\
\tau_{x y}
\end{array}\right]=\frac{E}{(1+\gamma)(1-2 \gamma)}\left[\begin{array}{cccccc}
1-\gamma & \gamma & \gamma & 0 & 0 & 0 \\
\gamma & 1-\gamma & \gamma & 0 & 0 & 0 \\
\gamma & \gamma & 1-\gamma & 0 & 0 & 0 \\
0 & 0 & 0 & \frac{(1-2 \gamma)}{2} & 0 & 0 \\
0 & 0 & 0 & 0 & \frac{(1-2 \gamma)}{2} & 0 \\
0 & 0 & 0 & 0 & 0 & \frac{(1-2 \gamma)}{2}
\end{array}\right]\left[\begin{array}{c}
\varepsilon_{x x} \\
\varepsilon_{y y} \\
\varepsilon_{z z} \\
\varphi_{y z} \\
\varphi_{z x} \\
\varphi_{x y}
\end{array}\right]
$$

$\left[\begin{array}{c}\varepsilon_{x x} \\ \varepsilon_{y y} \\ \varepsilon_{z z} \\ \varphi_{y z} \\ \varphi_{z x} \\ \varphi_{x y}\end{array}\right]=\left[\begin{array}{c}\varepsilon_{m, x x} \\ \varepsilon_{m, y y} \\ \varepsilon_{m, z z} \\ \varphi_{m, y z} \\ \varphi_{m, z x} \\ \varphi_{m, x y}\end{array}\right]-\left(T-T_{\mathrm{ref}}\right)\left[\begin{array}{c}\alpha \\ \alpha \\ \alpha \\ 0 \\ 0 \\ 0\end{array}\right]$

where $\gamma$ is the Poisson ratio, $\alpha$ is the thermal expansion coefficient and $E$ is the elasticity modulus. The strain displacement relations are

$\varepsilon_{m, x x}=\frac{\partial r_{x}}{\partial x}, \quad \varepsilon_{m, y y}=\frac{\partial r_{y}}{\partial y}, \quad \varepsilon_{m, z z}=\frac{\partial r_{z}}{\partial z}$

$\varphi_{m, x y}=\frac{\partial r_{x}}{\partial y}+\frac{\partial r_{y}}{\partial x}, \quad \varphi_{m, y z}=\frac{\partial r_{z}}{\partial y}+\frac{\partial r_{y}}{\partial z}, \quad \varphi_{m, z x}=\frac{\partial r_{z}}{\partial x}+\frac{\partial r_{x}}{\partial z}$

where $r_{x}, r_{y}$ and $r_{z}$ are the displacements. 
Eqs. (1)-(4) are nondimensionalized by using $D$ as the length scale, and constructing dimensionless velocities in the form of Reynolds numbers

$(\widetilde{x}, \widetilde{y}, \widetilde{z}, \widetilde{n})=(x, y, z, n) / D, \quad \widetilde{u}=u D / v, \quad \widetilde{v}=v D / v, \quad \widetilde{w}=w D / v$

The dimensionless pressure difference is defined as [32,33]

$\widetilde{P}=\frac{\left(P-P_{\text {out }}\right) D^{2}}{\mu \lambda}$

where $P, P_{\text {out }}, \mu$ and $\lambda$ are the local pressure, the outlet pressure, the dynamic viscosity, and the thermal diffusivity of the fluid $\left(k_{f} / \rho c_{P}\right)$. The dimensionless temperature and continuity of the heat flux are indicated by

$\widetilde{T}=\frac{\left(T-T_{\text {ref }}\right) k_{f}}{q^{\prime \prime} D}$

$\left.\widetilde{k} \frac{\partial \widetilde{T}}{\partial \widetilde{n}}\right|_{s}=\left.\frac{\partial \widetilde{T}}{\partial \widetilde{n}}\right|_{f}$

where $T_{\text {ref }}$ is the fluid inlet temperature and $\widetilde{k}=k_{s} / k_{f}$. Eqs. (7)-(11) are nondimensionalized by using $D$ as the length scale for displacements and coordinates, and $P_{m}$ as the pressure scale for the stresses, elasticity and mechanical load and by constructing the dimensionless thermal expansion coefficient $\widetilde{\alpha}$,

$\left(\widetilde{r}_{x}, \widetilde{r}_{y}, \widetilde{r}_{z}, \widetilde{x}, \tilde{y}, \widetilde{z}\right)=\left(r_{x}, r_{y}, r_{z}, x, y, z\right) / D$

$\widetilde{\varepsilon}_{m, x x}=\frac{\partial \widetilde{r}_{x}}{\partial \widetilde{x}}, \quad \widetilde{\varepsilon}_{m, y y}=\frac{\partial \widetilde{r}_{y}}{\partial \widetilde{y}}, \quad \widetilde{\varepsilon}_{m, z z}=\frac{\partial \widetilde{r}_{z}}{\partial \widetilde{z}}$

$\widetilde{\varphi}_{m, x y}=\frac{\partial \widetilde{r}_{x}}{\partial \widetilde{y}}+\frac{\partial \widetilde{r}_{y}}{\partial \widetilde{x}}, \quad \widetilde{\varphi}_{m, y z}=\frac{\partial \widetilde{r}_{z}}{\partial \widetilde{y}}+\frac{\partial \widetilde{r}_{y}}{\partial \widetilde{z}}, \quad \widetilde{\varphi}_{m, z x}=\frac{\partial \widetilde{r}_{z}}{\partial \widetilde{x}}+\frac{\partial \widetilde{r}_{x}}{\partial \widetilde{z}}$

$\left(\widetilde{E}, \widetilde{\sigma}, \widetilde{\tau}, \widetilde{P}_{l}\right)=\left(E, \sigma, \tau, P_{l}\right) / P_{l}$

$\widetilde{\alpha}=\frac{\alpha q^{\prime \prime} D}{k_{f}}$

where $P_{l}=10^{5} \mathrm{~N} \mathrm{~m}^{-2}$ is the pressure applied uniformly from the bottom of the plate. The dimensionless mass conservation and momentum equations are

$\frac{\partial \widetilde{u}}{\partial \widetilde{x}}+\frac{\partial \widetilde{v}}{\partial \widetilde{y}}+\frac{\partial \widetilde{w}}{\partial \widetilde{z}}=0$

$\widetilde{u} \frac{\partial \widetilde{u}}{\partial \widetilde{x}}+\widetilde{v} \frac{\partial \widetilde{u}}{\partial \widetilde{y}}+\widetilde{w} \frac{\partial \widetilde{u}}{\partial \widetilde{z}}=-\frac{1}{\operatorname{Pr}} \frac{\partial \widetilde{P}}{\partial \widetilde{x}}+\nabla^{2} \widetilde{u}$

$\widetilde{u} \frac{\partial \widetilde{v}}{\partial \widetilde{x}}+\widetilde{v} \frac{\partial \widetilde{v}}{\partial \widetilde{y}}+\widetilde{w} \frac{\partial \widetilde{v}}{\partial \widetilde{z}}=-\frac{1}{\operatorname{Pr}} \frac{\partial \widetilde{P}}{\partial \widetilde{y}}+\nabla^{2} \widetilde{v}$

$\widetilde{u} \frac{\partial \widetilde{w}}{\partial \widetilde{x}}+\widetilde{v} \frac{\partial \widetilde{w}}{\partial \widetilde{y}}+\widetilde{w} \frac{\partial \widetilde{w}}{\partial \widetilde{z}}=-\frac{1}{\operatorname{Pr}} \frac{\partial \widetilde{P}}{\partial \widetilde{z}}+\nabla^{2} \widetilde{w}$

where Pr is Prandtl number. The dimensionless energy equation is

$\operatorname{Pr}\left(\widetilde{u} \frac{\partial \widetilde{T}}{\partial \widetilde{x}}+\widetilde{v} \frac{\partial \widetilde{T}}{\partial \widetilde{y}}+\widetilde{w} \frac{\partial \widetilde{T}}{\partial \widetilde{z}}\right)=\nabla^{2} \widetilde{T}$

The dimensionless momentum equation and Hooke's law for the solid domain are

$\frac{\partial \widetilde{\sigma}_{x x}}{\partial \widetilde{x}}+\frac{\partial \widetilde{\tau}_{y x}}{\partial \widetilde{y}}+\frac{\partial \widetilde{\tau}_{z x}}{\partial \widetilde{z}}=0$ $\frac{\partial \widetilde{\tau}_{x y}}{\partial \widetilde{x}}+\frac{\partial \widetilde{\sigma}_{y y}}{\partial \widetilde{y}}+\frac{\partial \widetilde{\tau}_{z y}}{\partial \widetilde{z}}=0$

$\frac{\partial \widetilde{\tau}_{x z}}{\partial \widetilde{x}}+\frac{\partial \widetilde{\tau}_{y z}}{\partial \widetilde{y}}+\frac{\partial \widetilde{\sigma}_{z z}}{\partial \widetilde{z}}=0$

$\left[\begin{array}{c}\tilde{\sigma}_{x x} \\ \tilde{\sigma}_{y y} \\ \tilde{\sigma}_{z z} \\ \tilde{\tau}_{y z} \\ \tilde{\tau}_{z x} \\ \tilde{\tau}_{x y}\end{array}\right]=\frac{\widetilde{E}}{(1+\gamma)(1-2 \gamma)}\left[\begin{array}{cccccc}1-\gamma & \gamma & \gamma & 0 & 0 & 0 \\ \gamma & 1-\gamma & \gamma & 0 & 0 & 0 \\ \gamma & \gamma & 1-\gamma & 0 & 0 & 0 \\ 0 & 0 & 0 & \frac{(1-2 \gamma)}{2} & 0 & 0 \\ 0 & 0 & 0 & 0 & \frac{(1-2 \gamma)}{2} & 0 \\ 0 & 0 & 0 & 0 & 0 & \frac{(1-2 \gamma)}{2}\end{array}\right]\left[\begin{array}{c}\widetilde{\varepsilon}_{x x} \\ \widetilde{\varepsilon}_{y y} \\ \widetilde{\varepsilon}_{z z} \\ \widetilde{\varphi}_{y z} \\ \widetilde{\varphi}_{z x} \\ \widetilde{\varphi}_{x y}\end{array}\right]$

$\left[\begin{array}{c}\widetilde{\varepsilon}_{x x} \\ \widetilde{\varepsilon}_{y y} \\ \widetilde{\varepsilon}_{z z} \\ \widetilde{\varphi}_{y z} \\ \widetilde{\varphi}_{z x} \\ \widetilde{\varphi}_{x y}\end{array}\right]=\left[\begin{array}{c}\widetilde{\varepsilon}_{m, x x} \\ \widetilde{\varepsilon}_{m, y y} \\ \widetilde{\varepsilon}_{m, z z} \\ \widetilde{\varphi}_{m, y z} \\ \widetilde{\varphi}_{m, z x} \\ \widetilde{\varphi}_{m, x y}\end{array}\right]-\widetilde{T}\left[\begin{array}{c}\widetilde{\alpha} \\ \widetilde{\alpha} \\ \widetilde{\alpha} \\ 0 \\ 0 \\ 0\end{array}\right]$

The boundary conditions are

$\tilde{r}_{x}, \widetilde{r}_{y}, \widetilde{r}_{z}=0$ at $x=0-D$ and $y=(-D / 2)-(+D / 2)$ and $z=0-H$ on the rim

$\widetilde{P}_{l}=1$ and $\tilde{q}^{\prime \prime}=1$ at $z=0$ on the bottom surface

$\widetilde{P}_{\text {max }}=10^{7}-10^{8}$ and $\widetilde{P}_{\text {ref }}=0$

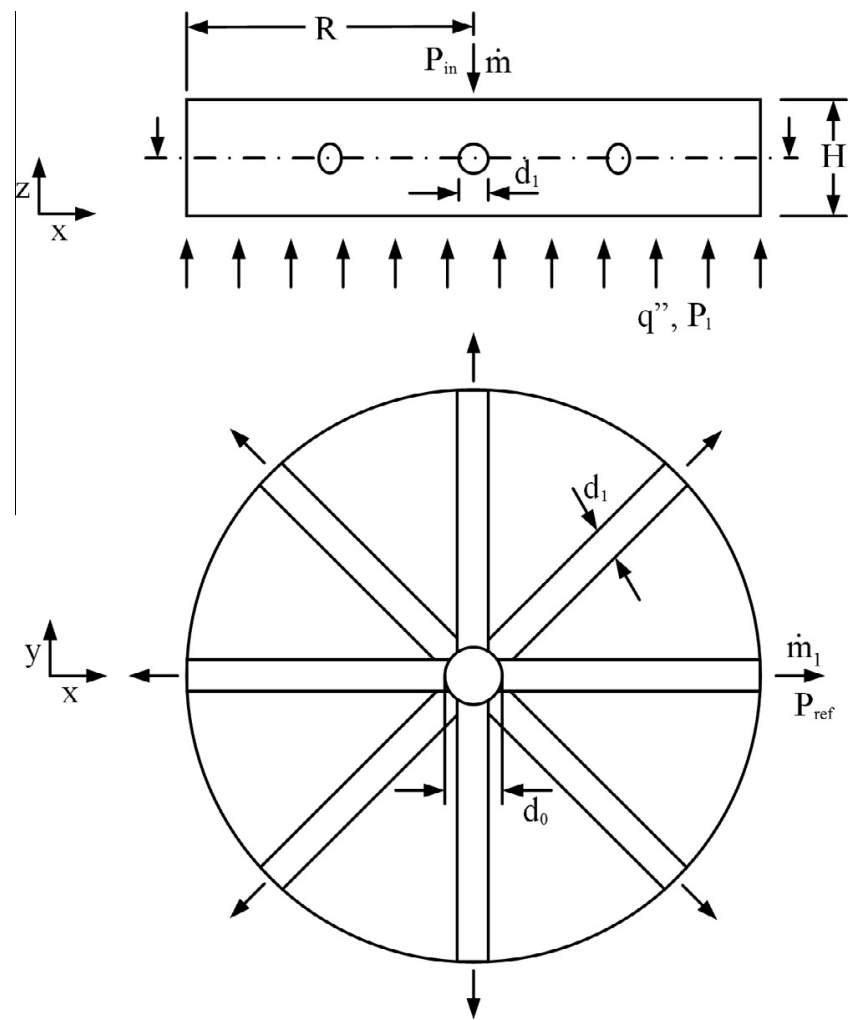

Fig. 1. Circular plate with embedded radial cooling channels. 


\section{Radial channels}

Consider the geometry of Fig. 1, which has cooling channels of one size $\left(d_{1}\right)$ connected to a distributing channel of diameter $d_{0}$ and length $L_{d}=0.07 D$. The bifurcations are accompanied with the Hess-Murray rule for laminar flow (problem 4.10 of Ref. [1])

$d_{0} / d_{1}=\eta^{1 / 3}$

where $\eta$ is the number of the cooling channels connected to the distributing channel. The pressure difference between the inlet and outlet is defined as [32,33].

$P_{\max }=\frac{\left(P_{\text {in }}-P_{\text {ref }}\right) D^{2}}{\mu \lambda}$

The outlet pressure $\left(P_{\text {ref }}\right)$ is 0 , while $P_{\max }$ is of order $10^{7}-10^{8}$. This $P_{\max }$ range corresponds to laminar flow, i.e. Reynolds numbers less than 2000 in all configurations. We also set $\widetilde{k}=20, \operatorname{Pr}=6$, $\gamma=0.33$ and $\widetilde{E}=2 \times 10^{6}$. The thermal expansion coefficient $\widetilde{\alpha}$ varied in the range $5 \times 10^{-6}-5 \times 10^{-4}$.

The dimensionless mass, momentum and energy equations were solved by using a finite element software [34]. The mesh was refined after each solution, and the problem was solved again to see whether the solution is mesh dependent. This was repeated by doubling the number of mesh elements until the results of the peak stress changed by less than $5 \%$.

The effect of the number of the cooling channels on the peak stress is shown in Fig. 2, where $V_{f}=0.03, P_{\max }=10^{8}$ and $\widetilde{\alpha}=10^{-4}$. Three curves are shown: stress due to only mechanical load (bottom), stress due to only thermal expansion (middle), and stress due to mechanical load and thermal expansion (top). Fig. 2 shows that thermal stresses decrease as the number of cooling channels increase, and that the stress due to mechanical load slightly decreases from $N=6$ to 8 and then increases from $N=8$ to 24 . Fig. 2 also shows that the superposition of mechanical and thermal stresses would yield incorrect result when the structure is heated and loaded mechanically, i.e. summation of the bottom and middle curves does not yield the top curve in Fig. 2. The reason of that is that the thermal expansion occurs in every direction, not only in one direction. Fig. 2 shows that peak stress in the circular plate becomes minimum when $N=8$ and 20 .

Fig. 3 shows how the peak stress varies relative to the dimensionless thermal expansion coefficient $\widetilde{\alpha}$ when $V_{f}=0.03, P_{\max }=10^{8}$ and $N=12$. The thermal stresses decrease as $\widetilde{\alpha}$ decreases, and become negligibly small relative to the mechanical stresses. Below $\widetilde{\alpha}=10^{-5}$, the effect of thermal expansion can be neglected. This result illustrates that the assumption of neglecting thermal stresses is valid when $\widetilde{\alpha}=10^{-5}$ and $\widetilde{P}_{l}=1$.

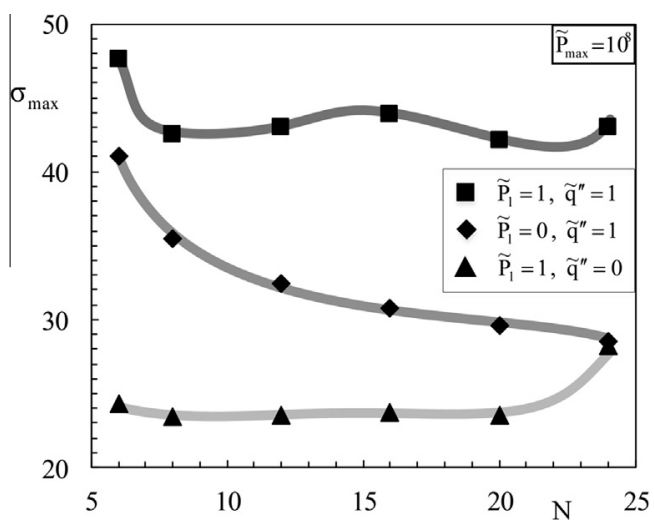

Fig. 2. The peak stress versus the number of radial cooling channels.

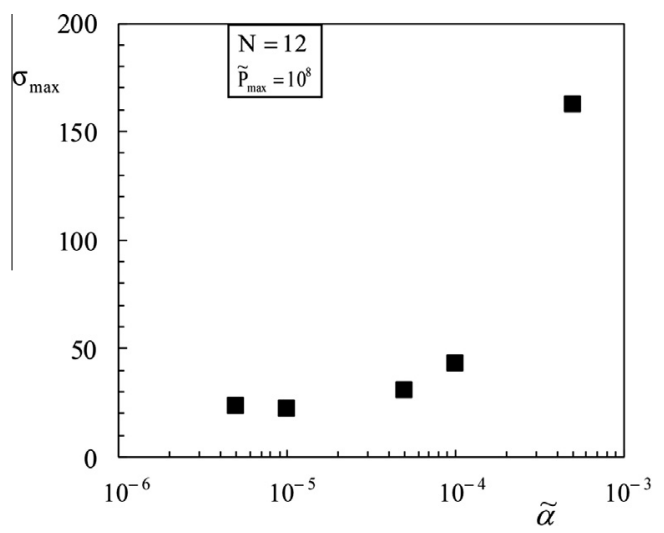

Fig. 3. The effect of the dimensionless thermal expansion coefficient on the peak stress.

Fig. 4a and b show how the peak stress and peak temperature change relative to the number of cooling channels and the volume fraction, at $P_{\max }=10^{8}$ and $P_{\max }=10^{7}$, respectively. The peak stress and peak temperature increase as $N$ increases when the flow volume is $1 \%$ of the solid volume, $V_{f}=0.01$. Fig. 4 a shows that the peak stress and the peak temperature decrease as $N$ increases when $V_{f}=0.03$ and $0.05, P_{\max }=10^{8}$. Fig. $4 \mathrm{~b}$ shows that the peak stress and peak temperature increase as $N$ increases for all three volume fractions when $P_{\max }=10^{7}$. The reason is that the coolant flow rate is not large enough to remove heat from the plate when $P_{\max }=10^{7}$ and $V_{f}=0.01$. As $P_{\max }$ and $V_{f}$ increase, the peak temperature and peak stress decrease, and after a certain level, increasing them does not change the result noticeably. For example, increasing $V_{f}$ from 0.03 to 0.05 when $P_{\max }=10^{8}$ does not change the order of the peak stress and peak temperature relative to increasing $V_{f}$ from 0.01 to 0.03 in Fig. 4a.

\section{Dendritic channels}

Next, consider Fig. 5, in which has three cooling channels of size $\left(d_{2}\right)$ are connected to a distributing channel of diameter $d_{0}$ and length $L_{d}=0.07 D$. The coolant enters from the center and is distributed through the first level of cooling channels of diameter $d_{2}$. At a junction, the channels bifurcate into two daughter channels of diameter $d_{3}$, which constitute the second level of cooling channels. The bifurcations are sized according to the Hess-Murray rule for laminar flow, cf. Eq. (36). The distance between outlet ports is fixed. The ratio of the flow volume to the solid volume is fixed at $V_{f}=0.03$. The pressure difference between the inlet and outlet ports is fixed at $P_{\max }=10^{8}$, which corresponds to laminar flow $(R e<2000)$ in all the cooling channels. The dimensionless thermal expansion coefficient $\widetilde{\alpha}$ is fixed at $10^{-4}$.

Fig. 6a shows how the peak stress changes relative to the length of the first level of channels, $L_{1}$, when the dendritic cooling channels have six outlet ports. There are two families of curves in Fig. 6a: the stress due to mechanical load (bottom) and the stress due to mechanical load and thermal expansion (top). The figure shows how the stress increases dramatically from the bottom curve to top curve when $\widetilde{\alpha}=10^{-4}$. The peak stress relative to $L_{1}$ seems as constant in Fig. 6a bottom curve, however, this is due to the limits of the ordinate axis. Fig. 6a shows that the peak stress becomes minimum and then maximum as $L_{1}$ increases. The reason of this behavior is because of the location on which peak stress occurs varies as $L_{1}$ increases. The peak stress is minimum when $L_{1}$ is equal to 0.15 with thermal expansion, and 0.3 without thermal expansion. 
(a)
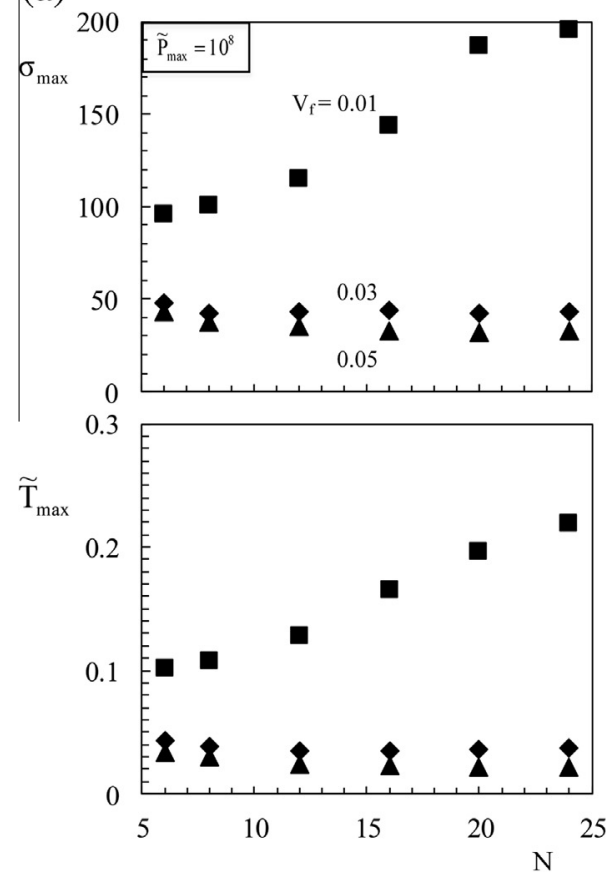

(b)
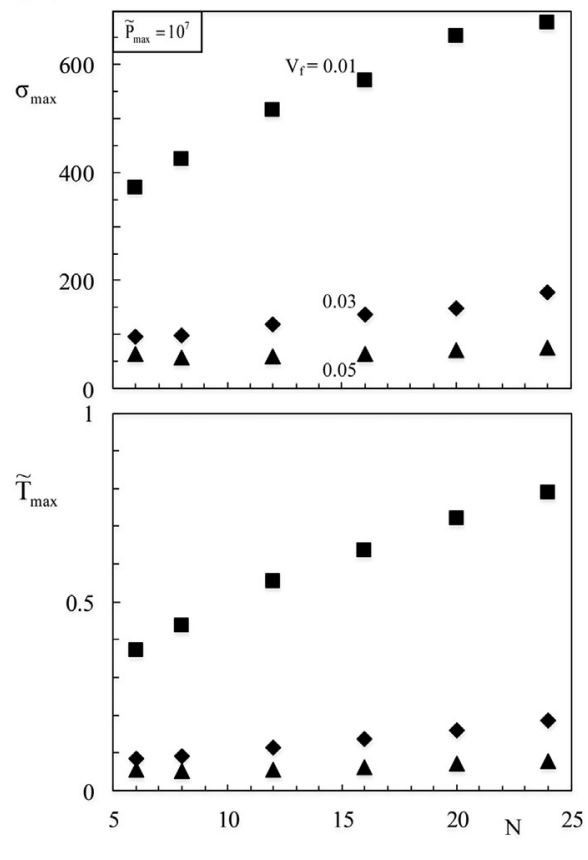

Fig. 4. The peak stress and peak temperature versus the number of radial cooling channels.

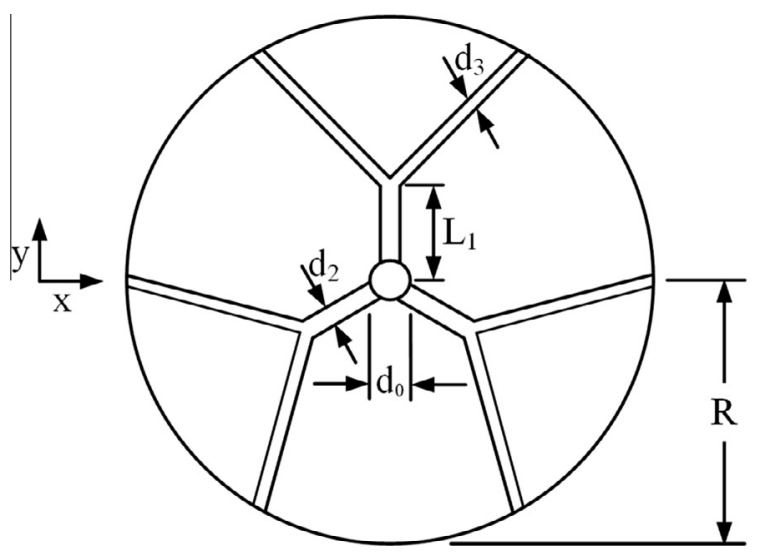

Fig. 5. Tree-shaped design with one pairing level.

Similarly, Fig. 6 b shows how the peak stress changes relative to the length of the first-level channels when there are eight outlet ports (i.e. four cooling channels of size $d_{2}$ are connected to the distributing channel of diameter $d_{0}$ ) and $\widetilde{\alpha}=10^{-4}$. The effect of the design parameter, $L_{1}$, becomes more significant in the presence of thermal expansion, as shown by the top curve in Fig. 6b. The peak stress increases as $L_{1}$ increases, and after a maximum at $L_{1}=0.15$, it decreases. The minimum peak stress is achieved when $L_{1}=0.3$ with and without thermal expansion.

Fig. 7 summarizes the effect of thermal stresses on the peak stress when a plate is heated and loaded mechanically. First, consider a circular plate with diameter $D$ and thickness $H_{s}$. This thickness is selected such as that the volume of this solid plate is equal to the vascularized plate when $V_{f}=0.03$, and $H_{s}<H$. The solid plate is subjected to mechanical load and heat flux as shown in Fig. 1. This solid plate is in contact with a heat sink at the center of the plate, and the heat sink surface area is selected as the area of the main distributing channel of diameter $d_{0}$ when $N=6$ as shown in

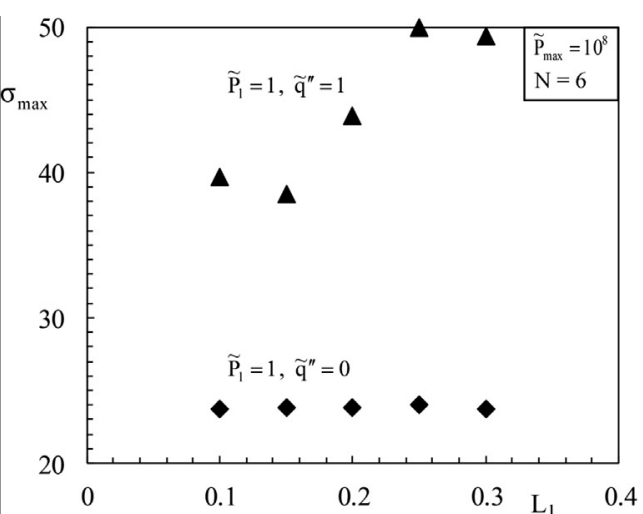

(a)

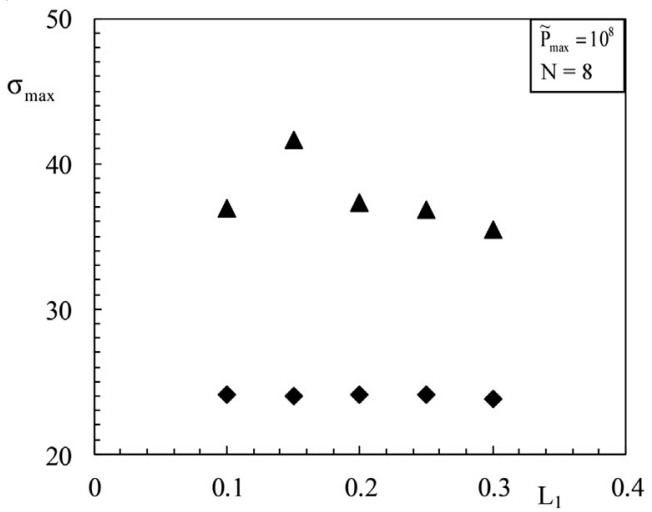

(b)

Fig. 6. The peak stress relative to the stem length $L_{1}$.

Fig. 1. Fig. 7 also shows the peak stresses of the radial and dendritic configurations. This figure has two parts: stress due to mechanical load (bottom) and stress due to mechanical load and thermal 

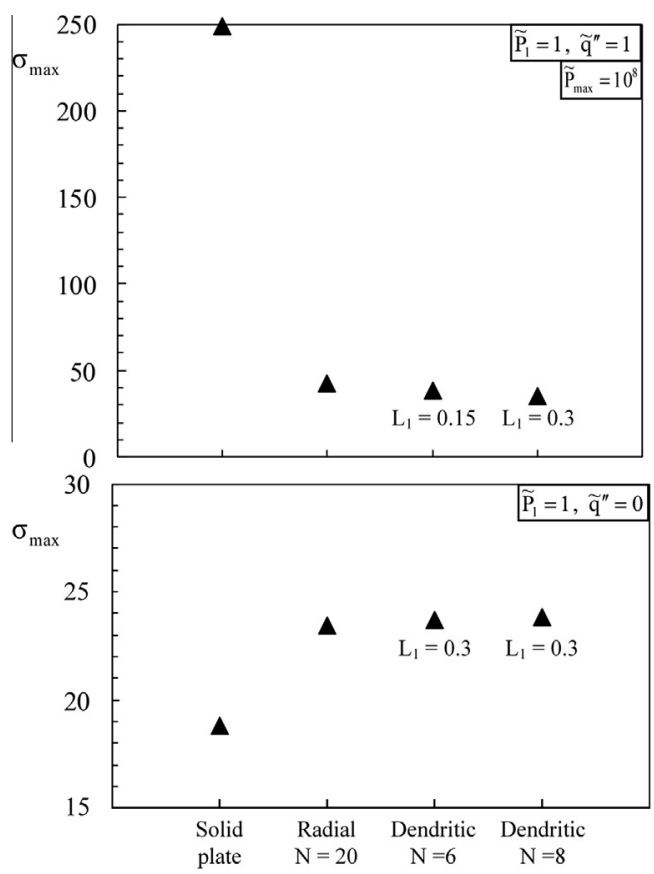

Fig. 7. The minimum peak stress versus design configuration: stresses due to mechanical load and thermal expansion (top), and stresses due only to mechanical load (bottom).

expansion (top). The peak stress increases because of embedding radial and dendritic designs, cf. Fig. 7 (bottom). Therefore, embedding a vascular cooling network decreases the strength of a plate when the thermal expansion effect is negligible, i.e. $\widetilde{\alpha}=10^{-5}$. However, the peak stress decreases when the effect of thermal expansion is considered, as shown in Fig. 7 (top). In addition, the effect of vascularization dramatically decreases the peak stresses, in addition to the peak temperatures. Changing the vascular cooling channels from radial to dendritic decreases the peak temperature but the effect diminishes as the number of the cooling channels increase, Fig. 7. In summary, embedding vascular cooling channels in a plate heated and loaded mechanically decreases the peak stress and peak temperature of the plate.

\section{Conclusions}

This paper showed that the thermal stresses and cooling performance of a circular plate can be improved simultaneously by embedding vascular cooling channels. The ratio of the flow volume over the solid volume was fixed. We explored the merits of the radial and dendritic cooling channels. We documented the configurations that performs the best for the given boundary conditions and constraints. One result is that increasing the volume fraction increases the mechanical strength and the cooling performance when the effect of thermal expansion is not negligibly small in comparison with the stresses due to the mechanical load. Mechanical strength and cooling performance improve as the design changes from radial to tree-shaped.

The chief conclusion is that when thermal stresses dominate the stress field the mechanical strength and cooling performance of a vascularized plate are significantly greater than in a solid plate without cooling channels. The effect of thermal expansion on the mechanical strength cannot be neglected when the dimensionless thermal expansion coefficient $\widetilde{\alpha}$ is greater than $10^{-5}$. In addition, a more uniform temperature distributions is achieved when vascularization is used.

\section{Conflict of interest}

None declared.

\section{Acknowledgments}

This work was supported by the National Science Foundation and the Republic of Turkey.

\section{References}

[1] A. Bejan, S. Lorente, Design with Constructal Theory, Wiley, Hoboken, 2008.

[2] K.-M. Wang, S. Lorente, A. Bejan, Vascularization with grids of channels: multiple scales, loops and body shapes, J. Phys. D Appl. Phys. 40 (2007) 47404749.

[3] A. Bejan, S. Lorente, K.-M. Wang, Networks of channels for self-healing composite materials, J. Appl. Phys. 100 (2006) 033528.

[4] A.M. Aragon, R. Saksena, B.D. Kozola, P.H. Geuebelle, K.T. Christensen, S.R. White, Multi-physics optimization of three-dimensional microvascular polymeric components, J. Comp. Phys. 233 (2013) 132-147.

[5] S. Soghrati, P.R. Thakre, S.R. White, N.R. Sottos, P.H. Geubelle, Computational modelling and design of actively-cooled microvascular materials, Int. J. Heat Mass Transfer 55 (2012) 5309-5321.

[6] J. Lee, Y. Kim, S. Lorente, A. Bejan, Constructal design of comb-like channel networks for self-healing and self-cooling, Int. J. Heat Mass Transfer 66 (2013) 898-905.

[7] E. Cetkin, S. Lorente, A. Bejan, Hybrid grid and tree structures for cooling and mechanical strength. J. Appl. Phys. 110 (2011) 064910.

[8] E. Cetkin, S. Lorente, A. Bejan, Vascularization for cooling and mechanical strength, Int. J. Heat Mass Transfer 54 (2011) 2774-2781.

[9] K.-H. Cho, J. Lee, H.S. Ahn, A. Bejan, M.H. Kim, Fluid flow and heat transfer in vascularized cooling plates, Int. J. Heat Mass Transfer 53 (2010) 36073614.

[10] L.A.O. Rocha, S. Lorente, A. Bejan, Tree-shaped vascular wall designs for localized intense cooling, Int. J. Heat Mass Transfer 52 (2009) 45354544.

[11] A. Bejan, S. Lorente, Constructal multi-scale and multi-objective structures, Int. J. Energy Res. 29 (2005) 689-710.

[12] S. Lorente, A. Bejan, Vascularized smart materials: designed porous media for self-healing and self-cooling, J. Porous Media 12 (2009) 1-18.

[13] E. Cetkin, Emergence of tapered ducts in vascular designs with laminar and turbulent flows, J. Porous Media, accepted for publication (2014)

[14] P. Xu, X.Q. Wang, A.S. Mujumdar, C. Yap, B.M. Yu, Thermal characteristics of tree-shaped microchannel nets with/without loops, Int. J. Therm. Sci. 48 (2009) 2139-2147.

[15] W. Wechsatol, S. Lorente, A. Bejan, Tree-shaped networks with loops, Int. J. Heat Mass Transfer 48 (2005) 573-583.

[16] L.A.O. Rocha, S. Lorente, A. Bejan, Conduction tree networks with loops for cooling a heat generating volume, Int. J. Heat Mass Transfer 49 (2006) 26262635.

[17] J. Yue, R. Boichot, L. Luo, Y. Gonthier, G. Chen, Q. Yuan, Flow distribution and mass transfer in a parallel microchannel contactor integrated with constructal distributors, AIChE J. 56 (2010) 298-317.

[18] L. Gosselin, Fitting the flow regime in the internal structure of heat transfer systems, Int. Commun. Heat Mass Transfer 33 (2006) 30-38.

[19] Y. Chen, C. Zhang, M. Shi, Y. Yang, Thermal and hydrodynamic characteristics of constructal tree-shaped minichannel heat sink, AIChE J. 56 (2010) 20182029.

[20] H. Zhang, S. Lorente, A. Bejan, Vascularization with line-to-line trees in counterflow heat exchange, Int. J. Heat Mass Transfer 52 (2009) 43274342.

[21] S. Muzychka, Constructal design of forced convection cooled microchannel heat sinks and heat exchangers, Int. J. Heat Mass Transfer 48 (2005) 31193127.

[22] Y.S. Muzychka, Constructal multi-scale design of compact micro-tube heat sinks and heat exchangers, Int. J. Therm. Sci. 46 (2007) 245-252.

[23] W. Wechsatol, S. Lorente, A. Bejan, Dendritic heat convection on a disc, Int. J. Heat Mass Transfer 46 (2003) 4381-4391.

[24] C. Zhang, Y. Chen, R. Wu, M. Shi, Flow boiling in constructal tree-shaped minichannel network, Int. J. Heat Mass Transfer 54 (2011) 202-209.

[25] G. Lorenzini, C. Biserni, L.A.O. Rocha, Constructal design of X-shaped conductive pathways for cooling a heat generating body, Int. J. Heat Mass Transfer 58 (2013) 513-520.

[26] S.K. Saha, M. Baelmans, A design method for rectangular microchannel counter flow heat exchangers, Int. J. Heat Mass Transfer 74 (2014) 1-12.

[27] M.R. Hajmohammadi, E. Shirani, M.R. Salimpour, A. Campo, Constructal placement of unequal heat sources on a plate cooled by laminar forced convection, Int. J. Therm. Sci. 60 (2012) 13-22.

[28] E. Cetkin, Three-dimensional high-conductivity trees for volumetric cooling, Int. J. Energy Res., published online, (2014), doi: 10.1002/er.3176.

[29] S. Lorente, A. Bejan, Combined "flow and strength" geometric optimization: internal structure in a vertical insulating wall with air cavities and prescribed strength, Int. J. Heat Mass Transfer 45 (2002) 3313-3320. 
[30] L. Gosselin, A. Bejan, S. Lorente, Combined 'heat flow and strength' optimization of geometry: mechanical structures most resistant to thermal attack, Int. J. Heat Mass Transfer 47 (2004) 3477-3489.

[31] S. Lorente, J. Lee, A. Bejan, The "flow of stresses" concept: the analogy between mechanical strength and heat convection, Int. J. Heat Mass Transfer 53 (2010) 2963-2968.
[32] S. Bhattacharje, W.L. Grosshandler, The formation of a wall jet near a high temperature wall under microgravity environment, ASME HTD 96 (1988) 711 716.

[33] S. Petrescu, Comments on the optimal spacing of parallel plates cooled by forced convection, Int. J. Heat Mass Transfer 37 (1994) 1283.

[34] See www.comsol.com for information about COMSOL Multiphysics. 